\title{
Traumatismo dental en pacientes pediátricos que acuden a una clínica universitaria de odontopediatría: un análisis retrospectivo de historias clínicas
}

\author{
Dental trauma in pediatric patients consulting at a university \\ pediatric dentistry clinic: a retrospective analysis of medical records
}

\author{
Ana Karen Garibay-Martínez', Norma Leticia Robles-Bermeo', Cesar Tadeo Hernández- \\ Martínez ${ }^{1,2}$, Luis Javier Guadarrama-Quiroz', Guadalupe Pedraza-Contreras' ${ }^{1}$, Sandra Isabel \\ Jimenez-Gayosso ${ }^{1,2}$, Carlo Eduardo Medina Solís ${ }^{1,2}$
}

\section{RESUMEN}

Objetivo: Determinar la prevalencia de traumatismos dentales en los pacientes que acuden a una clínica de Especialidad en Odontopediatría. Materiales y Métodos: Se realizó un estudio transversal retrolectivo, se incluyeron 309 expedientes clínicos de pacientes de 2 a 12 años de edad que acudieron para su atención a la clínica de la Especialidad de Odontopediatría de una universidad pública en Toluca, México. La variable dependiente fue la prevalencia de traumatismo dental. Las variables independientes incluidas en el estudio fueron: edad del paciente y sexo. El análisis estadístico se realizó utilizando pruebas no paramétricas en Stata 11. Resultados: El promedio de edad fue de $5.71 \pm 2.43$ años y el $50.8 \%$ fueron hombres. La prevalencia de traumatismo dental previo al estudio fue de $12.0 \%$ (IC $95 \%=8.3-15.6$ ). En el análisis bivariado, a pesar de que los varones presentaron una prevalencia de traumatismo dental de $14.0 \%$ y entre las mujeres fue de $9.9 \%$, estas diferencias no fueron estadísticamente significativas $(\mathrm{p}=0.262)$. Se observó que los niños con traumatismo dental fueron más pequeños (promedio de edad $=4.74 \pm 1.86$ ) que los que no presentaron traumatismo dental (promedio de edad=5.84 +2.47$)(\mathrm{p}=0.0166)$. Al realizar la prueba de tendencia no paramétrica se notó que la disminución de la prevalencia de traumatismo dental fue significativa ( $p=0.026$ ). Conclusiones: La prevalencia de traumatismo dental en esta muestra de niños fue de $12 \%$. No se observó diferencia por sexo. Los más pequeños presentaron más traumatismo dental que los más grandes.

Palabras clave: Salud bucal; traumatismo dental; niños; odontopediatría.

\begin{abstract}
Objective: To determine the prevalence of dental trauma in patients who consulted at a pediatric dentistry specialty clinic. Materials and methods: This was a retrospective cross-sectional study in which we reviewed 309 clinical files of patients from 2 to 12 years of age who consulted at the Pediatric Specialty Dentistry at a public university in Toluca, Mexico. The dependent variable was the prevalence of dental trauma. The independent variables included in the study were: patient's age and sex. Statistical analysis was performed using nonparametric tests in Stata 11. Results: The average age was $5.71 \pm 2.43$ years and $50.8 \%$ were male. The prevalence of dental trauma was $12.0 \%(95 \% \mathrm{CI}=8.3$ - 15.6). In the bivariate analysis, despite the fact that males had a prevalence of dental trauma of $14.0 \%$ and among women it was $9.9 \%$, these differences were not statistically significant $(\mathrm{p}=$ 0.262 ). It was observed that children with dental trauma were younger (average age $=4.74 \pm 1.86$ ) than those who did not present dental trauma (average age $=5.84 \pm 2.47)(\mathrm{p}$ $=0.0166)$. When performing the non-parametric trend test, it was noted that the decrease in the prevalence of dental trauma was significant $(p=0.026)$. Conclusions: The prevalence of dental trauma in this sample of children was $12 \%$. No difference was observed according to sex. Younger patients presented more dental trauma than older ones.
\end{abstract}

Keywords: Oral health; dental trauma; children; pediatric dentistry.

\footnotetext{
${ }^{1}$ Universidad Autónoma del Estado de México, Facultad de Odontología, Centro de Investigación y Estudios Avanzados en Odontología "Dr. Keisaburo Miyata". Toluca, México.

${ }^{2}$ Universidad Autónoma del Estado de Hidalgo, Instituto de Ciencias de la Salud, Área Académica de Odontología. Pachuca, México.

Correspondencia: Carlo Eduardo Medina-Solís; Correo: cemedinas@yahoo.com

Conflicto de interés: Los autores declaran no tener conflicto de intereses.
}

Recibido: 27/10/2018; Aceptado: 22/11/2018

DOI: https://doi.org/10.31698/ped.45032018003 


\section{INTRODUCCIÓN}

La caries dental es el principal problema de salud pública bucal en México y el mundo debido a la prevalencia e incidencia que se observa entre los niños en edad prescolar y escolar. A su vez, presentan altas necesidades de tratamiento para esta enfermedad y es la causa principal de consulta al dentista $^{(1-3)}$. En este sentido, el trauma dental, es también un motivo de atención dental en odontopediatría y en un futuro muy cercano, la incidencia de las lesiones por traumatismo dental constituirán un importante porcentaje de la demanda de atención dental ${ }^{(4)}$. Los traumatismos dentales generalmente no son una emergencia que amenace la vida y, por esa razón, pueden ser percibidos como una condición menos urgente por parte del público, así como del personal de los departamentos de emergencia. Sin embargo, se ha encontrado que la demora en proporcionar la atención adecuada pone en peligro significativamente el resultado del tratamiento y causa más complicaciones $^{(5)}$. El trauma dental se caracteriza porque no se rige por un sólo mecanismo etiopatogénico, ni sigue un patrón predecible en cuanto a la intensidad o extensión ${ }^{(4)}$, es una lesión causada por la transmisión aguda de la energía de un impacto sobre los dientes y/u otros tejidos duros y blandos dentro y alrededor de la boca y la cavidad oral. Por lo general, es repentino, circunstancial, inesperado, accidental y a menudo requiere atención de emergencia. No es una enfermedad, sino una consecuencia de varios factores de riesgo inevitables en la vida ${ }^{(6,7)}$. Se han descrito en la literatura seis tipos de lesiones en los tejidos perirradiculares (es decir, lesiones por luxación), siete tipos de fracturas dentales y sus combinaciones. Las respuestas de los tejidos determinarán el tratamiento requerido y el resultado/consecuencia de los dientes involucrados ${ }^{(7)}$. Pero la carga que tiene en los sistemas de salud y en las familias es alto. Incluso, en algunos países, el costo directo (tratamiento) e indirecto (pérdida de productividad y salarios, y gasto en transporte) del trauma se estima que es alrededor de 4 a 5\% del producto interno bruto, además de los costos intangibles asociados (pérdida de calidad de vida del paciente y del entorno familiar, sufrimiento, angustia y dolor ${ }^{(8)}$. Igualmente, el tratamiento implica costos económicos en el corto plazo (restauración de la dentadura dañada) y en el largo plazo (asistencia y renovación de la rehabilitación protésica).

Recientes revisiones han explorado la ocurrencia de trauma dental en diferentes regiones del mundo y revelaron que aproximadamente el $30 \%$ de los niños en edad preescolar han sufrido un traumatismo dental en la dentición primaria y el $25 \%$ de los escolares han sufrido un trauma dental que involucra la dentición permanente ${ }^{(9)}$. De igual forma, se ha informado una gran variación de su incidencia dentro y entre los países ${ }^{(10-14)}$. La variación de la prevalencia del trauma dental entre los estudios puede atribuirse a la ubicación geográfica, las disparidades ambientales y socioeconómicas, los criterios diagnósticos utilizados, así como las diferencias en las muestras estudiadas ${ }^{(9)}$. Otras revisiones mencionan que la incidencia de trauma dental va de 1 a 44 nuevos casos por cada 1000 personas en un año. $Y$ las cifras de prevalencia en el mundo pueden ir de $6 \%$ hasta $59 \%{ }^{(6)}$. El trauma dental es el resultado de la compleja interacción entre los factores ambientales, el comportamiento humano y las características orales ${ }^{(15)}$. Los estudios epidemiológicos indican que los varones tienen mayor riesgo de sufrir trauma dental. Y en cuanto a la edad, aunque ha sido estudiada en las investigaciones, permanece controversial $^{(15)}$.

Por todo lo anterior ya es considerado un problema de salud pública entre niños y adolescentes por diversos autores ${ }^{(10-14)}$. En México no existen antecedentes de estudios que identifiquen cifras sobre este problema. Con la hipótesis que existen diferencias del traumatismo por edad y sexo, el objetivo del presente estudio fue determinar la prevalencia de traumatismos dentales y su distribución por edad y sexo en pacientes que acuden a una clínica de Especialidad en Odontopediatría.

\section{MATERIAL Y MÉTODOS}

\section{Diseño, población y muestra}

Se realizó un estudio de diseño observacional, transversal, retrolectivo en pacientes de 2 a 12 años 
de edad. El método de selección de la muestra fue por muestreo no probabilístico. Parte de la metodología ha sido previamente publicada ${ }^{(16)}$. El cálculo del tamaño de muestra se realizó con un nivel de confianza del $95 \%$, una precisión de $4 \%$, una proporción (valor aproximado del parámetro) de $90 \%$ y un porcentaje esperado de pérdidas del $10 \%$, lo que resultó en 314 sujetos. Los criterios de inclusión de las historias clínicas fueron: a) de individuos de ambos sexos; b) que presentaran todos los elementos de diagnóstico; c) de 2 a 12 años de edad; d) autorizadas por los asesores de la clínica para su tratamiento; e) que tuvieran la firma de consentimiento informado. Los criterios de exclusión fueron: a) que presentaran datos confusos o que no se hubiera recabado correctamente la información; b) donde los padres no hubiesen autorizado la toma de fotografías clínicas. Finalmente, la muestra incluyó un total de 309 historias clínicas de pacientes que acudieron para su atención a la clínica de la Especialidad de Odontopediatría de la Universidad Autónoma del Estado de México entre enero de 2014 a diciembre de 2015. Dicha institución se localiza en la ciudad de Toluca de Lerdo, capital del Estado de México, y es la universidad pública del Estado. Brinda servicios especializados a bajo costo para la población en general, específicamente para ciudadanos de la región de todos los niveles socieoconómicos, aunque generalmente acuden los de nivel socioeconómico bajo y medio.

\section{Recolección de la información y variables}

En primera instancia se obtuvo la autorización de la Coordinación del Programa. Todos los datos se obtuvieron de las historias clínicas que previamente fueron respondidas por los padres y/o tutores de los pacientes. La variable dependiente fue la prevalencia de traumatismo dental, la cual se obtuvo de la pregunta: ¿su hijo ha sufrido de algún traumatismo o golpe en los dientes anteriormente? $0=\mathrm{No}, 1=\mathrm{Si}$. Las variables independientes incluidas en el estudio fueron: edad del paciente (de 2 a 12 años $)$ y sexo ( 0 = Masculino, 1 = Femenino $)$.

\section{Análisis estadístico}

Para realizar el análisis de los datos se empleó el paquete estadístico Stata 11.0. En el análisis univariado se reportan frecuencias y porcentajes para las variables cualitativas; así como medias y desviación estándar para las variables cuantitativas. Se generaron tablas para presentar los resultados.

Con la finalidad de buscar diferencias entre la prevalencia de traumatismo dental y las variables sexo y edad, se realizó un análisis bivariado. En éste se emplearon las pruebas de Mann Whitney (para traumatismo dental vs edad) y chi cuadrada (para traumatismo dental vs sexo), de acuerdo a la escala de medición de las variables que fueron contrastadas. Se consideró estadísticamente significativo un valor de $\mathrm{p}<0.05$.

\section{Consideraciones éticas}

El protocolo de estudio se aprobó por el comité de ética e investigación del Centro de Investigación y Estudios Avanzados en Odontología "Dr. Keisaburo Miyata" de la Facultad de Odontología de la Universidad Autónoma del Estado de México. La realización de la presente investigación cumplió con las especificaciones de la Ley General de Salud en materia de investigación y con los principios científicos de Helsinki. No se requirió la firma de consentimiento informado debido a que sólo se revisaron los expedientes clínicos.

\section{RESULTADOS}

En total se incluyeron 309 niños entre 2 y 12 años de edad, de los cuales $50.8 \%$ fueron hombres. El promedio de edad en la muestra fue de $5.71 \pm 2.43$ años (Cuadro 1). La prevalencia de traumatismo dental fue de $12.0 \%$ (IC 95\% $=8.3-15.6)(n=37)$.

Cuadro 1. Resultados descriptivos de las variables del estudio.

\begin{tabular}{|l|c|c|}
\hline \multirow{3}{*}{ Sexo } & Frecuencia & Porcentaje \\
\cline { 2 - 3 } Hombre & & \\
Mujer & 157 & 50.8 \\
\hline \multirow{3}{*}{ Edad } & 152 & 49.2 \\
\cline { 2 - 3 } & Promedio & DE \\
\hline
\end{tabular}

En el análisis bivariado de chi cuadrada (Cuadro 2), a pesar que los varones presentaron una prevalencia de traumatismo dental mayor $(14.0 \%)$ que las mujeres $(9.9 \%)$, estas diferencias no fueron 
estadísticamente significativas $(p=0.262)$. En el análisis de Mann-Whitney se observó que los niños con traumatismo dental fueron más pequeños (promedio de edad $=4.75 \pm 1.87$ ) que los que no presentaron traumatismo dental $(5.84 \pm 2.48)$ (Cuadro 2), esta diferencia fue estadísticamente significativa ( $p=0.0166)$. En un análisis por grupos de edad, se muestra que los de 2 a $<6$ años tuvieron la mayor prevalencia que los otros dos grupos de mayor edad $(\mathrm{p}=0.070)$. $\mathrm{Y}$ al realizar la prueba de tendencia no paramétrica se notó que la disminución de la prevalencia de traumatismo dental fue significativa $(\mathrm{p}=0.026)$.

Cuadro 2. Análisis bivariado del traumatismo dental por edad y sexo.

\begin{tabular}{|c|c|c|c|}
\hline & Sin traumatismo & Con traumatismo & Valor de $p$ \\
\hline $\begin{array}{l}\text { Sexo } \\
\text { Hombre } \\
\text { Mujer }\end{array}$ & $\begin{array}{l}135(86.0) \\
137(90.1)\end{array}$ & $\begin{array}{l}22(14.0) \\
15(9.9)\end{array}$ & $0.262^{*}$ \\
\hline Edad (años) & $5.84+2.48$ & $4.75+1.87$ & $0.0166+$ \\
\hline $\begin{array}{c}\text { Edad (categorías) } \\
2 \text { a }<6 \text { años } \\
6 \text { a }<8 \text { años } \\
8 \text { a } 12 \text { años }\end{array}$ & $\begin{array}{l}159(85.0) \\
48(88.9) \\
65(95.6)\end{array}$ & $\begin{array}{l}28(15.0) \\
6(11.1) \\
3(4.4)\end{array}$ & $\begin{array}{l}0.070 * \\
0.026 \neq\end{array}$ \\
\hline
\end{tabular}

* Chi cuadrada

+ Mann-Whitney

‡ Prueba de tendencia no paramétrica

\section{DISCUSIÓN}

Este estudio se propuso identificar el porcentaje de traumatismo dental. Así, la prevalencia de niños con traumatismo dental en esta muestra de pacientes que acudieron a una clínica especializada en odontopediatría en una universidad pública fue del $12 \%$. Esta cifra es similar a la reportada por FreireMaia et al., ${ }^{(13)}(14 \%)$ en su estudio realizado en Brasil en niños de 8 a 10 años; más baja que la observada en niños de 3 a 5 años por Sulieman y Awooda ${ }^{(14)}(18 \%)$ en Sudan, y en Irán por Eslamipour et al., ${ }^{(11)}$ (23.8\%) en niños de 9 a 14 años; pero más alta que en el estudio realizado en Turquía por Tumen et al., ${ }^{(12)}$ (4.6\%) en niños de 8 a 12 años, y en Reino Unido por Blokland et al., ${ }^{(10)}$ (9\%) en sujetos de 8 a 15 años. Sin embargo, es necesario destacar que los intervalos de edad no son exactamente iguales a los incluidos en este estudio. Lam $^{6}$ menciona que las diferencias en las cifras del trauma dental alrededor del mundo pueden ser dadas por el diseño de los estudios.
Igualmente reflejan diferencias locales, culturales, ambientales y socioeconómicas. Además, otra fuente metodológica de variabilidad entre los estudios es la manera en que se clasifican las lesiones. Feliciano y de Franca ${ }^{(17)}$ identificaron más de 50 sistemas de clasificación para el trauma dental. Estos sistemas de clasificación tienen diferencias en la definición, el alcance y los criterios de inclusión. Esto pone en relieve la importancia de estandarizar los reportes y sistemas de clasificación.

En la literatura mundial se informa que las lesiones dentales traumáticas ocurren con mayor frecuencia en los primeros años de vida cuando los niños comienzan a caminar y socializar, la causa subyacente más común en estos casos es una caída accidental $^{(18)}$. En este estudio notamos que los niños de menor edad fueron los más afectados. Se debe tomar en cuenta que otros estudios ${ }^{(12,13)}$ observan mayor prevalencia de traumatismos dentales entre los sujetos más grandes, sin embargo, nosotros incluimos un espectro de edad más amplio (de 2 a 12 años). Por lo que estas prevalencias deben estar cambiando de acuerdo a los grupos de edad en comparación, como se observó en el análisis de tendencia no paramétrica y tal como Vettore et al., menciona; que es más común el traumatismo en la dentición primaria que en la permanente.

Respecto al sexo, alrededor del mundo se reporta de manera consistente que los varones presentan mayor prevalencia de traumatismo dental ${ }^{(13,1,19)}, \sin$ embargo en este estudio, a pesar que se observó esta situación, la diferencia no fue estadísticamente significativa. Esto puede deberse a la incorporación de las niñas a actividades que antes estaban únicamente reservadas para niños en nuestro contexto.

Dentro de las limitaciones que tiene el estudio y que es necesario resaltar están los relacionados a su diseño transversal retrolectivo. Por ejemplo, estudios donde se utilizan datos secundarios (historias clínicas) la información se ve limitada a la disponibilidad de datos en los documentos consultados, por lo que un análisis sobre las causas, tratamiento y consecuencias del trauma dental se ve seriamente restringido. Para Lam ${ }^{(6)}$, el utilizar este tipo de estudios puede subestimar las verdaderas 
cifras de trauma dental. A pesar de esta situación, se recomienda realizar estudios epidemiológicos en fuentes no epidemiológicas. Otra limitación que se debe resaltar, es que no se pudo establecer en que dentición y que dientes específicos fueron los más afectados. Todo lo anterior genera una oportunidad para realizar cambios en las historias clínicas de las clínicas de odontopediatría y se pueda profundizar en el conocimiento del evento estudiado. Esto debido a que alrededor del mundo, las implicaciones sobre la salud y financieras que tiene, se le considera un problema de salud pública bucal ${ }^{(10-14,20-23) \text {. }}$

\section{CONCLUSIONES}

La prevalencia de traumatismo dental en esta muestra de niños fue de $12 \%$. No se observó diferencia por sexo. Los más pequeños presentaron más traumatismo dental que los mayores. El presente estudio aportó datos sobre la prevalencia de traumatismos en los pacientes pediátricos que acuden a una clínica especializada en odontopediatría. Es necesario realizar más estudios con mejor diseño para monitorear el comportamiento de los traumas dentales en otras comunidades de México, identificar factores de riesgo y proponer protocolos para su prevención y tratamiento.

8. Andersson L. Epidemiology of traumatic dental injuries. J Endod 2013;39:2-5.

9. Vettore MV, Efhima S, Machuca C, Lamarca GA. Income inequality and traumatic dental injuries in 12-year-old children: A multilevel analysis. Dent Traumatol. 2017;33(5):375-382.

10. Blokland A, Watt RG, Tsakos G, Heilmann A. Traumatic dental injuries and socioeconomic position findings from the Children's Dental Health Survey 2013. Community Dent Oral Epidemiol. 2016;44(6):586-591.

11. Eslamipour F, Iranmanesh P, Borzabadi-Farahani A. Cross-sectional Study of Dental Trauma and Associated Factors Among 9- to 14-year-old Schoolchildren in Isfahan, Iran. Oral Health Prev Dent. 2016;14(5):451-457.

12. Tumen EC, Yavuz I, Kaya S, Uysal E, Tümen DS, Ay Y, et al. Prevalence of traumatic dental injuries and associated factors among 8 to 12-years-old schoolchildren in Diyarbakir, Turkey. Niger J Clin Pract. 2017;20(10):12591266.

13. Freire-Maia FB, Auad SM, Abreu MHNG, Sardenberg F, Martins MT, Paiva SM, et al. Prevalence of and factors associated with enamel fracture and other traumas in Brazilian children8-10 years old. Braz Oral Res. 2018;32:e89.

14. Sulieman AG, Awooda EM. Prevalence of Anterior Dental Trauma and Its Associated Factors among Preschool Children Aged 3-5 Years in Khartoum City, Sudan. Int J Dent. 2018;2018:2135381. 
15. Aswathikutty A, Marcenes W, Stansfeld SA, Bernabé E. Obesity, physical activity and traumatic dental injuries in adolescents from East London. Dent Traumatol. 2017;33(2):137-142.

16. Hernández-Martínez CT, Robles-Bermeo NL, LaraCarrillo E, Medina-Solís CE, Jiménez-Gayosso SI, PontigoLoyola AP, et al. Factors associated with dental caries in primary and permanent dentition in patients aged 2 to 12 years who attended a university clinic. West Indian Med J 2018; 67:en prensa. doi: 10.7727/wimj.2017.120

17. Feliciano K, de Franca C. A systematic review of the diagnostic classifications of traumatic dental injuries. Dent Traumatol 2006;22:71-76.

18. Paredes V, Paredes C. Traumatismos dentarios en la infancia. An Pediatr Contin. 2005;3(6):375-8.

19. Tsuchiya S, Tsuchiya M, Momma H, Sekiguchi T, Kuroki K, Kanazawa K, et al. Factors associated with sports-related dental injuries among young athletes: a cross-sectional study in Miyagi prefecture. BMC Oral
Health. 2017;17(1):168.

20. AlZoubi F, Mannocci F, Newton T, Manoharan A, Djemal S. What do dental students know about trauma? Dent Traumatol. 2015;31(6):482-6.

21. Antunes LA, Rodrigues AS, Martins AM, Cardoso ES, Homsi N, Antunes LS. Traumatic dental injury in permanent teeth: knowledge and management in a group of Brazilian school teachers. Dent Traumatol. 2016;32(4):269-73.

22. Bilder L, Margvelashvili V, Sgan-Cohen H, Kalandadze M, Levin L, Ivanishvili R, et al. Traumatic dental injuries among 12- and 15-year-old adolescents in Georgia: results of the pathfinder study. Dent Traumatol. 2016;32(3):16973.

23. Magno MB, Neves AB, Ferreira DM, Pithon MM, Maia LC. The relationship of previous dental trauma with new cases of dental trauma. A systematic review and metaanalysis. Dent Traumatol. 2019;35(1):3-14 . 